\title{
Socialismo a la venezolana: cuestiones estructurales*
}

\section{Raúl González Fabre, SJ'}

Palabras clave: Venezuela, socialismo, política rentista, nacionalización de empresas.

Key words: Venezuela, Socialism, Nationalization.

En su incansable búsqueda de nuevos campeones del socialismo, la izquierda radical europea se ha unido a la latinoamericana en adoptar a Hugo Chávez como sólido candidato al título. El indudable carisma comunicacional del líder venezolano, junto con su antiamericanismo vocinglero y su buena disposición a financiar apoyos dondequiera que éstos se le ofrezcan, bastan a estas personas y grupos para compensar detalles como su trayectoria militar golpista, su acoso a las organizaciones sociales y los medios de comunicación que no domina, o su control sin resquicios de los sistemas electoral y judicial en Venezuela, que permite augurarle una larga estancia en el poder.

La justificación de esta querencia por Hugo Chávez suele componer dos elementos en principio legítimos: en él se busca tanto un gobernante preocupado por los pobres, a menudo burlados por las élites latinoamericanas, como una esperanza para quienes querrían ordenamientos sociales alternativos al actual estado de cosas en la economía global. El primer elemento predomina quizás entre los seguidores latinos de Chávez, mientras el segundo es más común entre sus admiradores europeos.

Por esa tendencia humana a representarnos cada situación a partir de la nuestra propia, no es raro que algunos proyecten en Chávez lo que sienten que les falta en casa, tomando palabras por realidades sin mucho indagar sobre el proceso venezolano en sí mismo. Quienes están

\footnotetext{
"Este artículo es una adaptación modificada para público no venezolano de otro publicado bajo el título "Socialismo a la venezolana: cinco problemitas" en la Revista SIC, Caracas (marzo 2007). El artículo se publica simultáneamente en Mensaje, revista de pensamiento social de los jesuitas de Chile, $\mathrm{n}^{\circ}$ 558, mayo de 2007, por acuerdo entre ambas revistas.
}

1 Instituto de Investigaciones Económicas y Sociales (IIES), Universidad Católica Andrés Bello (UCAB), Caracas (Venezuela). 
más interesados por lo que ocurre o se planea en Venezuela que por el valor simbólico del chavismo para otros propósitos, querrán saber en qué consiste en realidad éste. Para contribuir a ese conocimiento, proponemos en este artículo dirigir la mirada hacia su lógica económica subyacente. No en vano, en su Prólogo de la Contribución a la Crítica de la Economía Política (1859), Karl Marx afirma:

En la producción social de su vida, los hombres contraen determinadas relaciones necesarias e independientes de su voluntad, relaciones de producción, que corresponden a una determinada fase de desarrollo de sus fuerzas productivas materiales. El conjunto de estas relaciones de producción forma la estructura económica de la sociedad, la base real sobre la que se levanta la superestructura jurídica y política y a la que corresponden determinadas formas de conciencia social. El modo de producción de la vida material condiciona el proceso de la vida social, política y espiritual en general. No es la conciencia del hombre la que determina su ser, sino, por el contrario, el ser social es lo que determina su conciencia ${ }^{2}$.

No es preciso estar de acuerdo con la tesis central de este pasaje para reconocer la conveniencia de examinar las prácticas económicas que se desarrollan y se fomentan desde el poder, a fin de desentrañar el significado verdadero de los discursos políticos que las acompañan. Un gobierno liberal que incrementara las regulaciones (como los ha habido en Europa), no sería en verdad liberal, y un gobierno socialista que facilite la explotación del trabajo por el capital privado (como se está viendo en China), no es socialista. Veamos entonces en qué consiste el 'socialismo a la venezolana' de Hugo Chávez, y cuáles son sus problemas estructurales.

\section{Socialismo rentista}

Hugo Chávez fue reelegido en diciembre de 2006 con más de $60 \%$ de los votos. Durante la campaña y después, aseguró que votar por él era apoyar un 'socialismo del siglo XXI' de contornos imprecisos. Al mismo tiempo, el núcleo de su campaña consistió en una expansión del gasto público que dio lugar a una fenomenal piñata populista, con el dinero y las importaciones corriendo con una abundancia que recordaba el primer periodo de su archirrival Carlos Andrés Pérez ${ }^{3}$.

Los pobres respondieron así, una vez más, a un reflejo sólidamente establecido en la cultura pública venezolana. Ese reflejo parte de la convicción de que Venezuela es un país rico, puesto que cuenta con petróleo, gas, hierro, aluminio y otros minerales en abundancia. Se trata de una

${ }^{2}$ http://www.ucm.es/info/bas/es/marx-eng/oel/mrxoel15.htm

${ }^{3}$ Carlos Andrés Pérez, del partido Acción Democrática, fue presidente de Venezuela en dos ocasiones (1974-1979 y 1989-1993). Su primer periodo, coincidente con la multiplicación de los precios del petróleo que siguió a la guerra del Yon Kippur, constituye el paradigma del populismo venezolano basado en la distribución masiva de renta petrolera (subsidios al consumo, gratuidad universal de los servicios, importaciones baratas, expansión del empleo público, créditos sin retorno a las empresas, corrupción galopante...). En su segundo periodo, con un país endeudado y bajos precios del petróleo, aplicó un programa de ajuste macroeconómico de inspiración neoliberal. El descontento social con los sacrificios exigidos por ese programa, siendo así que Pérez había sido elegido sobre la expectativa de volver a la abundancia de su primer periodo, motivó dos intentos de golpe de Estado en 1992, el primero de ellos liderado por Hugo Chávez. A fin de evitar un tercer golpe inminente, la Corte Suprema lo depuso a petición del Congreso en mayo de 1993, cuando faltaba casi un año para el fin de su periodo presidencial. 
concepción premoderna de la riqueza de las naciones, que la hace consistir en su tesoro más que en sus capacidades productivas y competitivas ${ }^{4}$. Los recursos del subsuelo que constituyen el 'tesoro' de Venezuela, son propiedad del Estado, al que la población asigna por misión fundamental repartir la renta que ellos generan. Quien prometa hacerlo de forma más creíble, obtiene el voto de los pobres (y de otros muchos), y queda comprometido con cada votante a hacerle llegar la parte que le toca. Por eso, en la campaña electoral Chávez ahorró discursos y se dedicó a repartir a manos llenas, sabiendo que no hay mejor promesa que la acompañada de un adelanto ${ }^{5}$. Para hacer más eficaz el mensaje, le añadió amenazas directas a los empleados públicos, contratistas del Estado, y otros agentes que dependen económicamente del favor gubernamental $^{6}$.

Hay sin embargo un problema: ni siquiera el comandante Chávez puede engañarse respecto a que recibir renta del Estado a cambio básicamente del voto, por un lado, y producir al máximo de la propia capacidad sin espíritu de lucro para el beneficio de la colectividad, por otro lado, son movimientos opuestos del alma humana. Si se reclutan los partidarios y votantes por el primer procedimiento, va a ser verdaderamente difícil ponerlos a funcionar en la segunda dirección.

De hecho, ya Adam Smith notó que la actitud de la clase rentista, acostumbrada a recibir sin trabajo ni cuidado, es contraria tanto a la iniciativa empresarial basada en el propio interés de la clase capitalista, como al esfuerzo por la supervivencia y el ascenso social de los trabajadores asalariados. Nadie se mete en las complicaciones y los riesgos de la inversión, o en los sudores de un empleo productivo, si puede resolver su problema económico con renta de la tierra, del Estado, o de quien sea.

Por ello, el profesor Asdrúbal Baptista, reconocido investigador de la estructura macroeconómica nacional, ha insistido en que el gran proyecto económico del siglo XX venezolano, consistente en utilizar la renta petrolera para realizar la primera acumulación de un sistema capitalista moderno, era internamente contradictorio. Tiene toda la razón. El empresariado puesto a régimen de renta petrolera (protección contra importaciones, contratos sin licitación, incentivos, sobreprecios, dólares preferenciales, préstamos sin retorno...) ya tiene ahí la ganancia hecha:

\footnotetext{
${ }^{4}$ Esto tiene su correlato estructural. En su mensaje de fin de año, el presidente del Banco Central de Venezuela (banco emisor oficial), informó que el $89,5 \%$ de las exportaciones venezolanas del 2006 fueron en hidrocarburos. Si se suman minerales e hidroelectricidad, el porcentaje pasa de $95 \%$. Estas industrias extractivas y sus auxiliares no emplean, en conjunto, más de 200.000 personas en un país de 26 millones de habitantes. El resto de la población activa, unos 12 millones de personas, resulta básicamente incapaz de colocar nada en los mercados internacionales.
}

${ }^{5}$ El gasto público en octubre 2006 fue $83 \%$ más alto que en octubre 2005 , en términos reales $(97 \%$ en términos nominales). Los funcionarios públicos, por su parte, recibieron en noviembre un bono equivalente a cuatro meses de salario. Las elecciones tuvieron lugar el 3 de diciembre.

\footnotetext{
${ }^{6}$ Por ejemplo, a comienzos de noviembre, el ministro de Energía y Minas y presidente de PDVSA, la petrolera estatal venezolana, habló a toda la gerencia alta y media de la empresa, diciendo entre otras cosas: "Aquí estamos apoyando a Chávez, que es nuestro líder, que es el líder máximo de ésta Revolución. Y vamos a hacer todo lo que tengamos que hacer para apoyar a nuestro Presidente. Y el que no se sienta cómodo con esa orientación, es necesario que le ceda su puesto a un bolivariano." La reunión era a puerta cerrada, pero un asistente grabó el discurso con un móvil y fue retransmitido por televisión. El domingo siguiente, en su programa semanal, el Presidente apludió estas palabras de su ministro, quien obviamente no fue sancionado, ni por el Ejecutivo ni por el poder judicial o el electoral. El discurso completo puede verse y leerse en: http://lubrio.blogspot.com/2006/1 1/el-discurso-de-rafael-ramrez-pdvsa-s.html
} 
no necesita ni desea salir a competir en mercados inciertos con agresivos colombianos, o con chinos que trabajan dieciséis horas al día. La renta que iba a alimentar el capitalismo endógeno venezolano, lo pasmó después del empujón inicial. Cuando llegó la hora de la verdad y disminuyó seriamente la renta, no había empresa privada competitiva, capaz de concurrir a mercados abiertos sin apoyo del Estado, donde apalancar el desarrollo.

El rentismo paralizó al capitalismo criollo, con el que al menos compartía la búsqueda del propio interés como motivación fundamental. Ahora el socialismo a la venezolana pretende convertir al rentista que él mismo cultiva con sus dádivas, campañas y distribuciones, en un socialista capaz no sólo de producir eficientemente sino de hacerlo persiguiendo el interés comunitario tanto al menos como el suyo propio. Donde el capitalismo rentista fue imposible por internamente contradictorio, el socialismo rentista fracasará con más razón. No habrá socialismo en Venezuela, sino otra gargantuesca distribución de renta a través de esquemas todavía menos productivos y capaces de crear modernidad económica que los del capitalismo rentista.

\section{Estatizando con un Estado que no funciona}

Chávez no echó a perder el Estado venezolano, sino que llegó al poder precisamente porque el Estado venezolano estaba echado a perder. De ser el instrumento de la sociedad para su modernización, nuestro Estado se convirtió en el principal obstáculo para esa modernización: un aparato grande, costoso, ineficiente, no sólo torpe sino también entorpecedor de las iniciativas sociales, cada vez más fracasado en sus responsabilidades básicas de garantizar seguridad, educación, salud e infraestructura. Esta es probablemente la clave del desprestigio del régimen anterior y del resentimiento de muchos venezolanos, tanto del pueblo como de las clases profesionales, contra las élites políticas y económicas que permitieron tal decadencia beneficiándose de ella.

El Estado venezolano del siglo XX se comprendía a sí mismo como distribuidor/inversor de renta petrolera con un propósito modernizador sobre el país. El Estado falló no tanto por mal diseño de los sucesivos proyectos modernizadores, sino por la existencia de un criterio de distribución subyacente, siempre el mismo, siempre distinto al que cada proyecto modernizador proponía. Ese criterio sigue siendo: la renta se distribuye según la conexión del que recibe con el que reparte. Esa conexión puede ser personal, de negocios o política, con frecuencia todo ello a la vez. El Estado venezolano es capaz de convertir cualquier plan modernizador en un festín de renta petrolera repartida según redes de vínculos particulares.

El chavismo parece estar consciente de esto. Por ello no utiliza sino más bien duplica el Estado en los diversos planes y 'misiones' (programas sociales), para cuya organización ha creado estructuras paralelas con fuerte presencia de la Fuerza Armada y del Estado Cubano. Pues bien, pese a este reconocimiento práctico de la ineficiencia del Estado venezolano, el diseño económico del 'socialismo del siglo XXI' tiene como eje la reestatización de las columnas vertebrales de la economía, esto es, las grandes industrias: comunicaciones, energía, minas e hidrocarburos, quizás más tarde también alimentación, transporte, construcción, turismo, banca, educación... declarándolas 'estratégicas', 'de seguridad nacional', 'de utilidad pública', o semejantes. 
Esto no deja de sorprender. Un Gobierno que no sabe qué hacer con las funciones sociales básicas del Estado venezolano y anda subarrendándolas a militares y cubanos, pretende asumir con el mismo Estado disfuncional operaciones económicas de gran calado que el sector privado está realizando razonablemente bien, con mayor o menor beneficio para sus accionistas pero sin costo para el sector público.

Lo que vendrá después de la nacionalización, en el término de pocos años, es sabido porque hay experiencia previa. Basta rebobinar la película al periodo anterior a las privatizaciones de los años '90. Las industrias estatizadas irán saliendo de la lógica empresarial de negocios, por la que se sostienen sobre sus propios pies. Gerentes, empleados, clientes, contratistas y proveedores, todos entenderán que la empresa ha cambiado de naturaleza: ya no está para producir beneficio a sus accionistas sino para distribuir renta petrolera entre los allí conectados. Cada uno intentará sacar su tajada: los gerentes engordarán sus cuentas afuera y cultivarán fidelidades políticas; los clientes exigirán 'tarifas sociales' congeladas; los empleados presionarán por blindaje sindical para sus puestos de trabajo y colocarán a sus familiares en la empresa; los proveedores venderán con sobreprecios de escándalo; y los contratistas se aliarán con quien haga falta adentro para obtener el negocio. Las relaciones particulares, sea familiares, económicas o políticas, pronto predominarán sobre las reglas más o menos racionales que el Estado-propietario pretenda imponer. La inversión y la productividad decaerán; vendrán déficits y endeudamientos.

Las empresas públicas y los entes descentralizados fueron decisivos en la quiebra económica del régimen anterior a Chávez, precisamente porque al formar parte de un Estado distribuidor de renta, resultaron incapaces de concebirse a sí mismos como negocios de largo plazo. Asombra que se replique un modelo económico que fracasó estruendosamente en mejores condiciones institucionales y de capacidad profesional que las que este Gobierno puede conseguir. Dada su peculiar estructura política, basada en la polarización y la confrontación entre los sectores modernos y los tradicionales de la sociedad, el chavismo sólo puede obtener capacidad profesional para gerenciar esas empresas pagándola a precio de oro (sea de la clase media a la que odia y que le odia, o de Cuba, si es que allí la hay y el Gobierno quiere seguir con la extranjerización del Estado). Y sólo puede responder a las expectativas rentistas de su base social respecto a las industrias nacionalizadas, destruyendo el modelo de negocios de éstas y convirtiendo lo que era fuente de beneficios para sus dueños (y de impuestos para el Estado) en sumideros del petróleo que ya se pierde abundantemente por numerosos canales internos y externos, sin dejar apenas capitalización ninguna para el país.

\section{Empresas sin empresarios, mercados sin mercaderes}

Si la columna vertebral del socialismo del siglo XXI va a ser un manojo de grandes empresas estatales, en torno a ellas y también a los ministerios, alcaldías, 'misiones', etc., se espera que florezcan todo tipo de 'empresas sociales', como proveedores, contratistas y subcontratistas suyos. No hay un solo modelo privilegiado, sino que conforme se ensaya y fracasa uno, se intenta otro. Cooperativas, empresas cogestionadas, empresas mixtas Estado-trabajadores, microempresas varias, comunas, 'empresas de producción social', 'productores independientes', 
han sido ya implementados en mayor o menor escala, con fracasos espectaculares en algunos casos y básicamente sin éxitos replicables dignos de mención. Esclarecidos ideológos de todos los socialismos dignos del nombre, llegan continuamente a Venezuela con nuevas ideas bajo el brazo, buscando captar la atención del Comandante para hacer su experimento social con carne y dinero del pueblo venezolano. Por supuesto, también quedarán empresas privadas, sobre todo si son de quienes no se han manifestado contra el Gobierno, quizás modificadas por ley para introducir alguna forma de comisariato político. Sobre esto habrá más novedades cuando el Ejecutivo, que ha recibido poderes de la Asamblea Nacional para legislar directamente sobre casi cualquier materia durante dieciocho meses, promulgue su corpus legal revolucionario.

Las nuevas formas empresariales del socialismo del siglo XXI comparten tres características principales: (i) dependen de financiamiento estatal, o bancario forzado por el Estado, para su formación; (ii) dependen de contratos del Estado o de empresas públicas para su sobrevivencia; y (iii) no se desea que tengan empresario en el sentido capitalista de la palabra, esto es, un sujeto con iniciativa y capacidad de riesgo que coordina los factores de producción y promueve como suyo el éxito de la empresa en el mercado, porque va a lucrarse de él. Se prefiere algún tipo de gestión asambleística de la unidad de producción, lo que cualquiera con mínima experiencia sabe que resulta mucho más complicado que la gestión empresarial ordinaria. Claro está, si la gestión asambleística falla como es de temer, siempre cabrá sustituirla por la gestión burocrática directa a cargo de funcionarios del Estado, igual que en Cuba, probablemente con los mismos espléndidos resultados.

Por otra parte, hay que decir que en cuanto modelo productivo, estas nuevas posibilidades no han sido acogidas con gran entusiasmo por la base trabajadora que ya tiene empleo formal. Con cierta razón, los trabajadores ven más probabilidades de que se les respeten sus derechos y estabilidad laboral si son directamente empleados por el Estado, que si pertenecen a una cooperativa contratista de una alcaldía o una empresa pública. Por ello piden que en caso de nacionalizar, se les estatice por completo. Otra cosa son los desempleados, quienes probablemente prefieran algún apoyo laboral del Estado a ninguno, y estén mejor dispuestos a integrar estos ensayos empresariales. Dado que en Venezuela ya hay serios problemas para mantener la disciplina laboral en empresas convencionales, las dificultades pueden ser mayores en estas nuevas unidades con mecanismos más complicados de decisión y sanción, pobladas además con trabajadores poco acostumbrados al empleo formal.

Estas empresas hiperdependientes del Estado, sin una verdadera vocación empresarial detrás, difícilmente alcanzarán competitividad como para valerse por sí mismas en mercados abiertos. En realidad, la competencia en que deberán participar será de tipo político por contratos con entes públicos de diversa naturaleza, como ya ocurría con muchas empresas privadas del antiguo 'capitalismo rentista'. Tales contratos serán otorgados como siempre, por conexiones familiares, políticas o por 'participación' económica del funcionario otorgante. Faltando esos contratos, las empresas quebrarán después de haber consumido el crédito inicial o el aporte del ministerio, como ya ha pasado con muchas cooperativas y con alguna empresa cogestionada. Más que sencillamente disolverse, si no tienen cosa mejor que hacer, los involucrados plantearán un problema social exigiendo su derecho socialista fundamental a que el Gobierno les mantenga. 
El socialismo del siglo XXI, sin embargo, no se agota en colgarse del Estado. También cabe que las nuevas unidades económicas no estatales, o sólo parcialmente estatales, produzcan directamente para el mercado. Será principalmente un mercado interno bien protegido por el Gobierno, porque sin una decidida visión empresarial en completo control de la empresa, no hay manera de alcanzar la competitividad internacional que otros sí están logrando en América Latina.

Al igual que la figura del empresario, el mercado también disgusta al socialismo del siglo XXI. Lo atestiguan los controles de divisas y precios, que ya empiezan a dar sus frutos de desabastecimiento y severos problemas para los productores. Ahora bien, los controles meramente entorpecen el mercado; más ambiciosa es la idea, pergeñada por los ideológos del Régimen, de reemplazarlo con trueque en base a fichas que sólo valdrán para ciertos tiempos y lugares, retrotrayéndonos así a una experiencia -la de una moneda de cuero distinta en cada aldea- que Occidente dejó atrás hace mil años, cuando se reabrieron los caminos al final de la Edad Oscura y volvieron a circular los metales. Como cualquier estudiante de primero de economía aprende, los mercados son tanto más eficientes en coordinar a los agentes económicos cuanto más abiertos, competitivos y fluidos resulten. Por eso Europa une sus economías en un solo gran mercado, y sus monedas en el euro. El socialismo del siglo XXI promete lo contrario: fragmentar el mercado y la moneda hasta el nivel local.

Obstaculizar la acción empresarial, segmentar los mercados en unidades locales para el intercambio sin afán de lucro ni mediación de dinero (raíz de todos los males), formar pseudoempresas sociales a las que otorgar contratos por criterios políticos, y demás iniciativas microeconómicas del socialismo del siglo XXI, tendrán consecuencias. Por una parte, reducirán el poder y la libertad de acción económica de todos los agentes no estatales, incluidos los pobres; por tanto, darán mayor control de la sociedad al Gobierno, de forma que prácticamente nadie pueda sobrevivir sin su aprobación, que es de lo que se trata. Por otra parte, incrementarán más aún la ineficiencia del sistema de producción-distribución, lo que será encubierto con renta petrolera para financiar importaciones masivas con que mantener el consumo ${ }^{7}$ y contentar a las bases sociales del Régimen, mientras se pueda. Después, probablemente vendrá la represión.

\section{Superestructura ideológica: el fantasma del Hombre Nuevo}

La respuesta del Régimen a estas obviedades es denunciar que semejante pronóstico se hace desde una mentalidad capitalista neoliberal, y que la educación socialista nos traerá el Hombre Nuevo, capaz de trabajar solidariamente por el colectivo, con independencia de lo que le toque en el reparto del producto. "De cada cual según su capacidad, a cada cual según sus necesidades", según el mismo Marx 8 .

7 El sector agropecuario es donde la revolución va más avanzada, con más de mil expropiaciones e invasiones de fincas privadas. Siguiendo a un desabastecimiento continuado de carne, pollo, azúcar, leche, arroz y otros víveres, a finales de febrero de 2007 el Gobierno anunció la importación de 990 mil toneladas de alimentos, esto es, casi cuarenta kilos de comida por habitante. La superficie de Venezuela es casi doble que la de España, para 15 millones de personas menos.

${ }^{8}$ En la Crítica del programa de Gotha (1875), I. 
Hay varios problemas aquí. Uno primero es que no aparecerá ese Hombre Nuevo al mismo tiempo laborioso y solidario, ni con la educación socialista ni con ninguna otra. La existencia humana adulta transcurre en medio de algunas tensiones constitutivas, que pueden ser vividas como creadoras o destructivas. La tensión entre individualidad y comunidad es una de ellas. La educación puede ayudar al joven a plantear tales tensiones de manera constructiva, pero no puede resolverlas por él. Esa es la tarea de la vida moral entera de cada persona. El Estado no puede sustituir en ello a la gente sin debilitar la sociedad en su mismo núcleo: la persona como sujeto moral que hace proyectos para sí y para el mundo. Si lo intentara, el resultado no sería la solidaridad, sino la parálisis social.

Esto viene confirmado por la experiencia histórica. El socialismo lleva 90 años gobernando países y construyendo el Hombre Nuevo desde el monopolio de la educación, la propaganda, los medios y la represión. Raymond Barre notaba en los años '70 que el 3\% de la tierra cultivada de la URSS que se dedicaba a huertos familiares, producía tanto como el otro $97 \%$ de las explotaciones colectivas en koljoses y sovjoses. Una vez desaparecido el régimen soviético, lo que había debajo resultó ser no el Hombre Nuevo sino la mafia del vodka. En China, el 'Gran Salto Adelante' mató de hambre a unos 30 millones de Hombres Nuevos, debidamente encuadrados en comunas populares autosuficientes. Por el contrario, el actual despegue económico comenzó a finales de los '70 con la reforma que permitió volver a la explotación privada de la tierra. Hoy casi tres cuartos del producto chino es generado en el sector privado. Más cerca de nosotros, Cuba ofrece otro ejemplo interesante: casi cincuenta años guiados por el Hombre Nuevo en persona, y no se ha conseguido el pleno abastecimiento de los productos básicos.

Construir un modelo socioeconómico sobre ficciones antropológicas resulta extraordinariamente costoso en tiempo, dinero, sangre y dignidad. Lo razonable es tomar las personas como ellas son en promedio, sin suponer que desde el poder político se pueden provocar extraordinarios cambios morales o culturales rápidamente. A partir de ahí, la sociedad debe organizarse de manera que unos con otros se inhiban las tendencias negativas, y se refuercen las positivas. El siglo XX ha mostrado claramente cómo se hace esto: hay que distribuir el poder tanto como sea funcional en el Estado y en la sociedad, de manera que el tono de la vida política venga dado por la negociación civilizada, el control mutuo y el respeto a las reglas acordadas. Y hay que establecer economías mixtas en que aproximadamente el 60-65\% del producto se gestione en mercados libres, abiertos y competitivos de bienes privados, y en torno al 35-40\% sea gestionado a través de un Gobierno democrático con fuertes controles políticos, judiciales y periodísticos, para la producción de bienes públicos, la conservación de recursos comunes, y cierta igualación de oportunidades.

Los detalles hay que adaptarlos a cada país, pero esa fórmula general ha sido probada con éxito en contextos europeos, americanos, latinos y asiáticos, en países muy distintos formados por personas normales, con las motivaciones y calidades morales usuales. En Venezuela se está haciendo precisamente lo contrario -concentrar el poder y cegar los precarios mercados existentes-, lo cual como es obvio no puede tener éxito con el venezolano normal, sino que se precisa uno novedosísimo, que actúe por motivaciones justamente opuestas a las que llevan a tanta gente ordinaria a votar por Chávez.

Toda la predicación sobre el Hombre Nuevo denota que este proyecto lleva el fracaso económico construido dentro: ya estamos viendo a Chávez una y otra vez luchando con ese fantasma, 
protestando que su misma gente no actúa como se necesita para el socialismo del siglo XXI, exigiendo un venezolano inexistente para el Estado que él quiere, en vez de preguntarse cómo ajustar el Estado que tiene al venezolano que hay, para hacer más funcionales a ambos. Al final concluirá, entre las ruinas de la nación, que no le entendimos y no le merecíamos.

\section{Superestructura gerencial: Gobernando por ocurrencias}

El problema central de la economía política probablemente sea cómo coordinar la acción de los innumerables agentes de una sociedad compleja, para que resulte en una gestión eficiente de los bienes escasos. En el capitalismo, esa coordinación ocurre por la iniciativa descentralizada de los agentes económicos, guiados por los precios que se forman impersonalmente en mercados abiertos y competitivos. En la teoría estándar del socialismo, el Estado asume sobre sí la tarea de coordinar a los agentes a través de una planificación centralizada: fija precios y cuotas de producción de muchos bienes y servicios, y asigna qué agentes deben producir y cuáles deben recibir cada cosa. Los mercados son marginales, y la planificación estatal se vuelve la clave de la economía.

Buena parte del fracaso del socialismo real estriba en que en sociedades complejas como las contemporáneas, con subjetividades individuales y colectivas bien desarrolladas, donde la creación cultural y la innovación tecnológica son decisivas para la generación de valor económico, la información precisa para realizar eficientemente esa planificación no está al alcance del Estado. No lo está primero porque el grueso de tal información es subjetiva; por tanto sólo cada agente conoce la suya propia, sin que pueda hacerse gran cosa para que la revele salvo organizar adecuadamente los incentivos. Además, la invasión de la economía por la lógica burocrática distorsiona continuamente la generación y transmisión de información, falseándola en cada nivel. Mienten los ciudadanos, mienten los funcionarios y mienten los políticos, cada uno según sus propósitos, sin controles horizontales eficaces como en las sociedades abiertas. Estos problemas son comunes a todos los sistemas de planificación central, pero en el caso del socialismo real demostraron ser fatales.

La planificación nunca ha sido una especialidad nacional, así que podríamos temer que lo que fracasó en la Alemania Oriental falle en Venezuela con más razón. No parece sin embargo que vaya a presentarse ese problema, porque el socialismo del siglo XXI no consiste en la planificación central de la economía sino en el gobierno por ocurrencias del Caudillo. Su eslógan no es 'poder de los soviets y electrificación de Rusia' como en Lenin, sino más bien: 'Comandante ordene, nosotros obedecemos', según rezaba una gran pancarta oficial puesta de fondo en el estrado donde Chávez anunció sus primeras medidas económicas tras la reelección. Esa es la técnica de coordinación económica del socialismo a la venezolana, muy semejante por cierto a la de los despotismos clásicos.

Ello no sólo se refiere a la relación entre Estado y sociedad, sino también a las relaciones dentro del mismo tren ejecutivo. Los ministros se enteran de muchas iniciativas que deben ejecutar en el mismo acto en que se anuncian al país, así que, todavía con las manos calientes de aplaudir, tienen que salir de urgencia a averiguar qué hacer con ello. En la mayor parte de los casos no 
hay mucho que pueda hacerse, y el resultado en materia económica es un cementerio de proyectos anunciados solemnemente, fallidos y abandonados un par de años después, tras haber enterrado en ellos algunos miles de millones.

El Comandante es hombre ocurrente, por lo que ordena esto y lo otro, según se va inspirando. Sus órdenes son en verdad personales: denotan una comprensión de la economía concentrada en el mundo campesino de producción semiartesanal del que él proviene. Quisiera llevar ese mundo a las hiperpobladas y hacinadas ciudades venezolanas, y por eso propone que la gente cultive huertos organopónicos en cualquier pedacito de tierra, o levante "gallineros verticales" en el techo de sus casas. No hemos tenido personalmente el gusto de ver el primero, ni de unos ni de los otros.

Chávez quisiera también sacar población de las ciudades y llevarla de vuelta al campo, no a la manera de Pol-pot sino más bien ensalzando las virtudes de la vida rural, y seduciendo con promesas de tierra, dinero y servicios. Para realizar esta idea, se propone construir nuevos núcleos campesinos en lugares remotos y deshabitados (que, obviamente, no lo están por casualidad). De esto hay también experiencia previa en Venezuela. Por razones de seguridad fronteriza, gobiernos anteriores ya intentaron crear nuevos poblados agrícolas en las fronteras con Colombia. Todos ellos fracasaron: ningunó creció más allá de la población inicial, y sus actuales habitantes son mayormente peones colombianos que trabajan en las haciendas de los alrededores. La frontera es ahora más insegura que nunca, controlada de hecho por guerrilleros, narcotraficantes y paramilitares.

Chávez parece no haber registrado estas experiencias de fracaso en la relocalización de población marginal urbana a zonas rurales. Los nuevos intentos cuya concreción ya ha anunciado, tienen aún menos probabilidades de éxito que los anteriores, porque el tiempo no pasa en vano. La población activa urbana ya no es la primera generación que vino del campo en los años ' 60 y' 70 , sino sus hijos y nietos, nacidos y criados en la ciudad, cuyas expectativas y habilidades están todas relacionadas con la sobrevivencia y el ascenso social en contextos urbanos. El intento de llevarlos 'de vuelta' a un campo en el que nunca han estado, fracasará a un costo económico muy alto.

Puesto que se trata de un Gobierno personalista, donde la palabra del Caudillo lo es todo y quien se atreva a discutirle algo pronto es excluído, los límites de la planificación económica del socialismo a la venezolana son los de la comprensión económica personal de Chávez. Según hemos señalado, esa comprensión abarca lo agropecuario en escala artesanal o semiartesanal, la pequeña producción doméstica, y las actividades extractivas. No incluye, sin embargo, ninguna visión realista sobre la producción de bienes y servicios en escala industrial, su comercialización en mercados competitivos, ni el desarrollo tecnológico, institucional y organizacional que ambas requieren. Al fin, antes de ser Presidente de la República, el cargo de gestión más importante que Chávez había desempeñado fue el de administrador de la cantina de su batallón.

Se trata de una perspectiva preocupante, se notará, para una sociedad compleja como la venezolana, con no menos de cincuenta años en contacto directo con la modernidad económica, la cual ha moldeado las aspiraciones de consumo, progreso económico y ascenso social de toda la población, también de los pobres. Justamente el fracaso en proporcionar caminos viables para 
satisfacer esas aspiraciones de modernidad económica, deslegitimó al régimen democrático anterior y abrió la puerta a la esperanza que muchos venezolanos han depositado en Chávez. Por ahora, esa esperanza se satisface con la distribución masiva de renta petrolera. Pero esta no es solamente un sucedáneo de modernidad económica sino que, como mencionamos arriba, constituye un mal sucédaneo, porque mueve a los agentes en la dirección opuesta a la requerida para valerse por sí mismos en economías modernas.

Por desgracia, no parece que Chávez tenga plan ninguno viable para revertir las tendencias rentistas de la sociedad venezolana y ofrecer verdadera modernidad económica a los pobres, en la que ellos puedan participar productivamente y de cuyos frutos puedan apropiarse. Más bien al contrario: las órdenes del Comandante trazan una economía estatizada sobre un Estado en derrumbe, con empresas sin empresarios y mercados sin mercaderes, que habrá que alimentar con importaciones masivas para evitar que se vacíen los anaqueles; una economía gestionada a partir de la improvisación de un Caudillo que piensa en términos de la Venezuela rural de hace medio siglo. Una economía cuya estructura última consiste en distribución de renta petrolera a cambio de obediencia política, dinero rodando sin esfuerzo ni riesgo para que el pueblo y los vividores del caso sigan siendo de Chávez.

El 'socialismo del siglo XXI' de Hugo Chávez sólo es sostenible en la medida en que los precios internacionales de los hidrocarburos le provean renta como para contentar a su base social, que en el caso de los pobres es lógicamente muy sensible al desabastecimiento de productos básicos y la inflación. Sólo en esa medida será posible disimular la inviabilidad de un modelo económico de extracción y distribución, sin verdadera propuesta productiva. Por ello, parece claro que no se trata de un "socialismo" reproducible fuera de los contados países de Latinoamérica que puedan literalmente 'vivir de la renta' 9 . Los primeros candidatos a seguir la vía venezolana han sido la Bolivia gasífera y el Ecuador petrolero. Probablemente sean también los únicos: no importa cuánto puedan identificarse ideológicamente con Chávez, ni Daniel Ortega ni Néstor Kirschner, por poner dos ejemplos, están en condiciones estructurales de imitarle. Otros líderes relevantes de la izquierda latinoamericana, como Lula da Silva, Michelle Bachelet, Oscar Arias, Alan García o Leonel Fernández, han dejado claro que ni siquiera se lo plantean.

Por su parte, la izquierda radical, tanto europea como latinoamericana, haría bien en aplicar su reconocida capacidad crítica a los discursos de Chávez, realizando la tarea marxista de analizar las relaciones económicas subyacentes a esos discursos, para evitar caer en una variante romántica del "doctrinarismo de izquierda" que Lenin denunció en otro contexto como síntoma de una enfermedad infantil del socialismo. Corrientes ideológicas que han gobernado países de todos los tamaños durante casi un siglo, y han podido ver las consecuencias de varias generaciones de propuestas suyas, no deberían estar ya para enfermedades infantiles.

\footnotetext{
${ }^{9}$ Curiosamente, los ideólogos oficiales del chavismo sostienen la teoría marxista del valor-trabajo. La izquierda radical que les acompañe en ello, debería notar que el dinero que Chávez reparte a manos llenas dentro y fuera de Venezuela, es el producto de la explotación del proletariado en las economías que compran petróleo a Venezuela. En efecto, si el trabajo es la única fuente legítima de valor económico, el valor que Venezuela se apropia vendiendo su petróleo muy por encima del costo de producción, ha de ser extraído de los trabajadores de otros países, en una manera semejante a como la burguesía utiliza los bienes de capital para enajenar plusvalía de los mismos trabajadores. La revolución chavista está entonces basada en la explotación del proletariado internacional, no menos que cualquier revolución burguesa.
} 